\title{
SOME PROPERTIES OF ABSOLUTELY MONOTONIC FUNCTIONS
}

\author{
P. C. ROSENBLOOM
}

In this note we collect several fragmentary results which were obtained as by-products of another investigation. They are rather loosely connected with each other, but still may be of some interest.

We recall that a function $f\left(x_{1}, \cdots, x_{k}\right)$ is said to be absolutely monotonic in a set $D$ if $f$ and all its partial derivatives exist and are non-negative in $D$. If $D$ is of the form $0 \leqq x_{i}<a_{i}, i=1, \cdots, k$, then a necessary and sufficient condition that $f$ be absolutely monotonic in $D$ is that it can be expanded in a power series in $x_{1}, \cdots, x_{k}$ with non-negative coefficients converging in $D$. (The well known theorem of Bernstein [1] $]^{1}$ for the case $k=1$ can be extended in a trivial manner.)

THEOREM 1. If $f(x)$ is absolutely monotonic in $0 \leqq x<a$, and if $0 \leqq x_{1}, x_{2}, \cdots, x_{n}<a$, and if $L(x)$ is the Lagrange interpolation polynomial of $f(x)$ at the points $x_{1}, \cdots, x_{n}$, then

$$
g(x)=\frac{f(x)-L(x)}{\omega(x)}, \quad \omega(x)=\left(x-x_{1}\right) \cdots\left(x-x_{n}\right),
$$

is an absolutely monotonic function of $x, x_{1}, \cdots, x_{n}$ in the range $0 \leqq x, x_{1}, \cdots, x_{n}<a$.

Proof. The function $g(x)$ can be expressed as a divided difference of $f(x)$ (see for example, Milne-Thompson [2]):

$$
g(x)=\left[x x_{1} \cdots x_{n}\right],
$$

where

and

$$
\left[x x_{1}\right]=\frac{f(x)-f\left(x_{1}\right)}{x-x_{1}}
$$

$$
\left[x x_{1} \cdots x_{k}\right]=\frac{\left[x x_{1} \cdots x_{k-1}\right]-\left[x_{k} x_{1} \cdots x_{k-1}\right]}{x-x_{k}}, \quad k=2, \cdots, n .
$$

It is sufficient, then, to show that if $f(x)$ is absolutely monotonic in $0 \leqq x<a$ then

Received by the editors August 7, 1945, and, in revised form, January 14, 1946.

${ }_{1}$ Numbers in brackets refer to the Bibliography at the end of the paper. 


$$
\frac{f(x)-f\left(x_{1}\right)}{x-x_{1}}
$$

is absolutely monotonic in the square $0 \leqq x, x_{1}<a$. But if $f(x)$ $=\sum_{n=0}^{\infty} a_{n} x^{n}, a_{n} \geqq 0$, then

$$
\frac{f(x)-f\left(x_{1}\right)}{x-x_{1}}=\sum_{n=1}^{\infty} a_{n}\left(x^{n-1}+x_{1} x^{n-2}+\cdots+x_{1}^{n-1}\right)
$$

is a power series with non-negative coefficients converging for $0 \leqq x$, $x_{1}<a$, and is therefore absolutely monotonic there.

Corollary. We have $f(x) \geqq L(x)$ for $x$ in the intervals $\left[x_{n}, a\right]$, $\left[x_{n-2}, x_{n-1}\right], \cdots$, while $f(x) \leqq L(x)$ in the intervals $\left[x_{n-1}, x_{n}\right],\left[x_{n-8}\right.$, $\left.x_{n-2}\right], \cdots$; if the equality sign holds at an interior point of any of these intervals, it holds identically.

It will be convenient to introduce the notations

$$
V\left(k_{1}, \cdots, k_{n}\right)=\left|\begin{array}{c}
x_{1}^{k_{1}} \ldots x_{1}^{k_{n}} \\
x_{n}{ }^{k_{1}} \ldots x_{n}^{k_{n}}
\end{array}\right|
$$

and

$$
A\left(k_{1}, \cdots, k_{n}\right)=\frac{V\left(k_{1}, \cdots, k_{n}\right)}{V(0,1, \cdots, n-1)} .
$$

LEMMA. If $0 \leqq k_{1}<k_{2}<\cdots<k_{n}$, where $k_{1}, \cdots, k_{n}$ are integers, then $A\left(k_{1}, \cdots, k_{n}\right)$ is a symmetric polynomial in $x_{1}, \cdots, x_{n}$, with nonnegative coefficients.

Proof. It is obvious that $A$ is a symmetric function. For $n=1$, $A\left(k_{1}\right)=x_{1} k_{1}$, and the lemma is true. Suppose now that $n>1$. Then setting $m_{i}=k_{i+1}-k_{1}$, we have

$$
\begin{aligned}
& A\left(k_{1}, \cdots, k_{n}\right)=x_{1}^{k_{1}} \cdots x_{n}^{k_{1}} A\left(0, m_{1}, \cdots, m_{n-1}\right)
\end{aligned}
$$

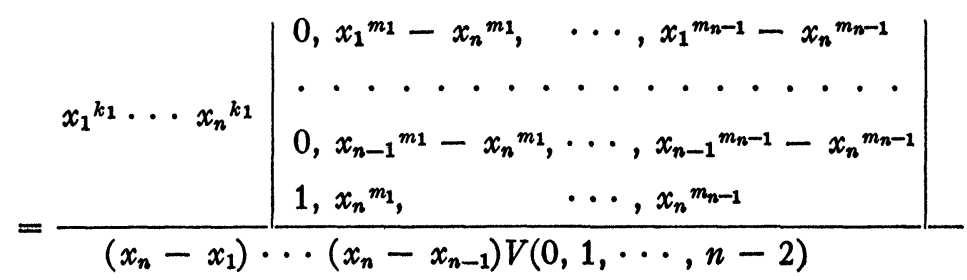

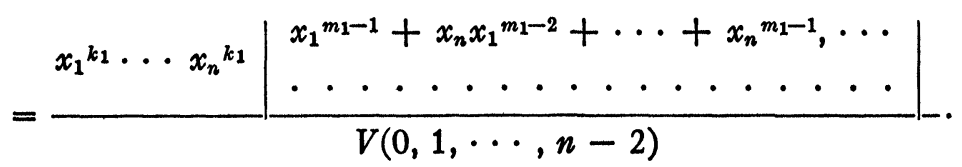


Now we multiply the first column in the numerator by $x_{n}{ }^{m_{2}-m_{1}}$ and subtract from the second; then we multiply the first column by $x_{n}{ }^{m_{3}-m_{1}}$ and the second by $x_{n}{ }^{m_{3}-m_{2}}$ and subtract from the third and so on. We thus obtain

$$
\begin{aligned}
& A\left(k_{1}, \cdots, k_{n}\right)=\frac{x_{1}^{k_{1}} \cdots x_{n}^{k_{1}}}{V(0,1, \cdots, n-2)} \\
& \left|\begin{array}{l}
x_{1}{ }^{m_{1}-1}+x_{n} x_{1}^{m_{1}-2}+\ldots+x_{n}{ }^{m_{1}-1}, x_{1}{ }^{m_{2}-1}+\ldots+x_{n}{ }^{m_{2}-m_{1}-1} x_{1}{ }^{m_{1}}, \ldots \\
\ldots \ldots \ldots \ldots \ldots \ldots \ldots \ldots \ldots \ldots \ldots \ldots \ldots \ldots \ldots \ldots \ldots \ldots
\end{array}\right| \\
& =x_{1}^{k_{1}} \cdots x_{n}{ }^{k_{1}} \sum x_{n}{ }^{\beta} A\left(\alpha_{1}, \cdots, \alpha_{n-1}\right) \text {, }
\end{aligned}
$$

where $A\left(\alpha_{1}, \cdots, \alpha_{n-1}\right)$ is formed with the indicated exponents and the variables $x_{1}, \cdots, x_{n-1}$, and the summation runs over all systems of $\alpha$ 's satisfying

$$
0 \leqq \alpha_{1} \leqq m_{1}-1, m_{1} \leqq \alpha_{2} \leqq m_{2}-1, \cdots, m_{n-2} \leqq \alpha_{n-1} \leqq m_{n-1}-1,
$$

and

$$
\beta=\left(m_{1}-1-\alpha_{1}\right)+\cdots+\left(m_{n-1}-1-\alpha_{n-1}\right) .
$$

Hence if the lemma is true for $n-1$, it is true for $n$. The lemma follows, then, by induction.

ThEOREM 2. If $f(x)$ and $g(x)$ are absolutely monotonic in the interval $0 \leqq x<a$, then

$$
\frac{1}{V(0,1,2)}\left|\begin{array}{lll}
1 & f\left(u x_{1}\right) & g\left(v x_{2} x_{3}\right) \\
1 & f\left(u x_{2}\right) & g\left(v x_{3} x_{1}\right) \\
1 & f\left(u x_{3}\right) & g\left(v x_{1} x_{2}\right)
\end{array}\right|
$$

is an absolutely monotonic function of its five arguments for $0 \leqq x_{1}, x_{2}$, $x_{3}<a, 0 \leqq u \leqq 1 / a, 0 \leqq v \leqq 1 / a^{2}$.

PROOF. Let $f(x)=\sum_{n=0}^{\infty} a_{n} x^{n}, g(x)=\sum_{n=0}^{\infty} b_{n} x^{n}, a_{n} \geqq 0, b_{n} \geqq 0, n=0$, $1, \cdots$. Then

$$
\begin{aligned}
\frac{1}{V(0,1,2)}\left|\begin{array}{ccc}
1 & u^{m} x_{1}{ }^{m} & g\left(v x_{2} x_{3}\right) \\
1 & u^{m} x_{2}{ }^{m} & g\left(v x_{3} x_{1}\right) \\
1 & u^{m} x_{3}{ }^{m} & g\left(v x_{1} x_{2}\right)
\end{array}\right| \\
=\sum_{n=0}^{\infty} \frac{b_{n}}{V(0,1,2)}\left|\begin{array}{llll}
1 & u^{m} x_{1}{ }^{m} & v^{n} x_{2}{ }^{n} x_{3}{ }^{n} \\
1 & u^{m} x_{2}{ }^{m} & v^{n} x_{3}{ }^{n} x_{1}{ }^{n} \\
1 & u^{m} x_{3}{ }^{m} & v^{n} x_{1}{ }^{n} x_{2}{ }^{n}
\end{array}\right| .
\end{aligned}
$$


Now

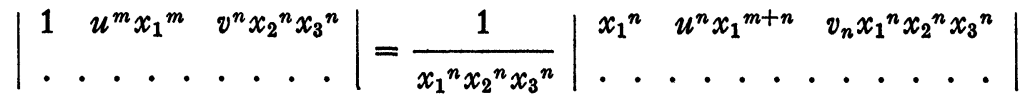

$$
\begin{aligned}
& =u^{m v^{n}} V(n, m+n, 0) \text {. }
\end{aligned}
$$

Hence

$$
\frac{1}{V(0,1,2)}\left|\begin{array}{lll}
1 & f\left(u x_{1}\right) & g\left(v x_{2} x_{3}\right) \\
1 & f\left(u x_{2}\right) & g\left(v x_{3} x_{1}\right) \\
1 & f\left(u x_{3}\right) & g\left(v x_{1} x_{2}\right)
\end{array}\right|=\sum_{m, n=0}^{\infty} a_{m} b_{n} u^{m^{n}} A(0, n, m+n) .
$$

If the terms of the last series are rearranged we obtain a power series in the five variables with non-negative coefficients converging in the range specified above.

TheOREM 3. If $f(x)=\sum_{n=0}^{\infty} a_{n} x^{n}, a_{n}>0$, and $a_{n+1} / a_{n}$ is monotonically non increasing, and if $f(x)$ and $g(x)$ are absolutely monotonic in $0 \leqq x<a$, then

$$
\frac{1}{V(0,1,2)}\left|\begin{array}{lll}
1 & f\left(x_{1}\right) & f\left(x_{1}\right) g\left(x_{1}\right) \\
1 & f\left(x_{2}\right) & f\left(x_{2}\right) g\left(x_{2}\right) \\
1 & f\left(x_{3}\right) & f\left(x_{3}\right) g\left(x_{3}\right)
\end{array}\right|
$$

is an absolutely monotonic function of all three variables for $0 \leqq x_{1}, x_{2}$, $x_{3}<a$.

Proof. Let $g(x)=\sum_{n=0}^{\infty} b_{n} x^{n}, b_{n} \geqq 0,0 \leqq x<a$. Let $0 \leqq x_{1}, x_{2}, x_{3} \leqq r<a$. Then

$$
\begin{gathered}
\frac{1}{V(0,1,2)}\left|\begin{array}{ccc}
1 & f\left(x_{1}\right) & f\left(x_{1}\right) g\left(x_{1}\right) \\
1 & f\left(x_{2}\right) & f\left(x_{2}\right) g\left(x_{2}\right) \\
1 & f\left(x_{3}\right) & f\left(x_{3}\right) g\left(x_{3}\right)
\end{array}\right| \\
\quad=\sum_{m=1}^{\infty} \sum_{n=0}^{\infty} \frac{a_{n} a_{m}}{V(0,1,2)}\left|\begin{array}{ccc}
1 & x_{1}{ }^{m} & x_{1}{ }^{n} g\left(x_{1}\right) \\
1 & x_{2}{ }^{m} & x_{2}{ }^{n} g\left(x_{2}\right) \\
1 & x_{3}{ }^{m} & x_{3}{ }^{n} g\left(x_{3}\right)
\end{array}\right| \\
=\sum_{m=1}^{\infty} \sum_{n+q=2}^{\infty} a_{n} a_{m} b_{q} A(0, m, n+q) .
\end{gathered}
$$

Now by elementary estimates

$$
\begin{aligned}
|A(0, m, n+q)| & \leqq 2^{-1} m(n+q)(m+n+q-2) r^{m+n+q-3} \\
& \leqq m^{2}(n+q)(n+q-1) r^{m+n+q-3}
\end{aligned}
$$

if $m \geqq 1, n+q \geqq 2$ and $\left|a_{n}\right| \leqq C R^{-n},\left|b_{n}\right| \leqq C R^{-n}$, where $r<R<a$, and $C$ is a suitably chosen constant. Then this series is dominated by 


$$
\begin{aligned}
C^{2} r^{-8} \sum_{m=1}^{\infty} \sum_{n+q=2}^{\infty} m^{2}(n+q)(n+q-1)(r / R)^{m+n+q} \\
=C^{2} r^{-3}\left(\sum_{m=1}^{\infty} m^{2}(r / R)^{m}\right)\left(\sum_{k=2}^{\infty} k(k+1)(k-1)(r / R)^{k}\right)
\end{aligned}
$$

and is therefore absolutely convergent. Hence we can rearrange it as follows:

$$
\begin{aligned}
\sum_{m=0}^{\infty} a_{m}\left\{-\sum_{\alpha=0}^{m-1} A(0, \alpha, m)\right. & \left.\sum_{q=0}^{\alpha} a_{\alpha-q} b_{q}+\sum_{\beta=m+1}^{\infty} A(0, m, \beta) \sum_{q=0}^{\beta} a_{\beta-q} b_{q}\right\} \\
& =\sum_{\alpha<\alpha<\beta} A(0, \alpha, \beta)\left\{\sum_{q=0}^{\infty} b_{q} a_{\alpha} a_{\beta}\left(\frac{a_{\beta-q}}{a_{\beta}}-\frac{a_{\alpha-q}}{a_{\alpha}}\right)\right\},
\end{aligned}
$$

and the latter can be rearranged as a power series in $x_{1}, x_{2}$, and $x_{8}$. But

$$
\frac{a_{\alpha-q}}{a_{\alpha}}=\frac{a_{\alpha-q}}{a_{\alpha-q+1}} \cdot \frac{a_{\alpha-q+1}}{a_{\alpha-q+2}} \ldots \cdot \frac{a_{\alpha-1}}{a_{\alpha}} \leqq \frac{a_{\beta-q}}{a_{\beta-q+1}} \ldots \cdot \frac{a_{\beta-1}}{a_{\beta}}=\frac{a_{\beta-q}}{a_{\beta}} .
$$

Therefore the resulting power series has only non-negative coefficients.

We feel that the last two theorems are at most mere curiosities as they stand, since we haven't any idea as to what significance the above determinants may have. It is to be hoped that proofs can be found which will be more illuminating than the above purely computational ones.

\section{BIBLIOGRAPHY}

1. S. Bernstein, Sur la définition et les proprietes des fonctions analytiques d'une variable rélle, Math. Ann. vol. 75 (1914) pp. 449-468.

2. L. M. Milne-Thomson, The calculus of finite differences, London, 1933.

BROWN UNIVERSITY 